\title{
Espesor medio intimal carotídeo en niños escolares y su relación con factores de riesgo cardiovascular
}

\author{
Alfonso Jáuregui *, Esperanza Fajardo, Jairo Castro y Yeimi Camargo
}

\author{
Universidad Militar Nueva Granada, Facultad de Medicina, Bogotá, Colombia
}

Recibido el 4 de junio de 2015; aceptado el 15 de octubre de 2015

Disponible en Internet el 24 de noviembre de 2015

\author{
PALABRAS CLAVE \\ Pediatría; \\ Factores de riesgo \\ cardiovascular; \\ Prevención primaria; \\ Arterias carótidas e \\ imágenes de \\ ecografía
}

\begin{abstract}
Resumen
Objetivo: Determinar la relación entre los factores de riesgo cardiovascular y el espesor medio intimal carotídeo en una población de escolares de la ciudad de Bogotá.

Método: Estudio descriptivo de corte transversal, al que fueron invitados todos los estudiantes de dos colegios seleccionados por conveniencia. La muestra correspondió a 94 escolares (57 niñas y 37 niños) de $13 \pm 1,54$ años. Se tomaron medidas antropométricas, presión arterial y medición ecográfica del espesor medio-intimal carotídeo. El análisis de datos se llevó a cabo mediante estadística descriptiva, en tanto que el análisis univariado y bivariado se hizo a través de tablas de distribución de frecuencias y tablas de contingencia para variables cualitativas y medidas descriptivas de tendencia central y de variabilidad para variables cuantitativas.

Resultados: Del total de la población, el 34,79\% presentó exceso de peso según el índice de masa corporal, el $28,8 \%$ registró exceso de grasa corporal, el $46 \%$ tuvo cifras tensionales elevadas de donde el 36,8\% correspondió a prehipertensión arterial y 9,2\% a hipertensión arterial estadio 1 . El promedio del espesor de la arteria carótida fue de 0,3163 $\pm 0,040 \mathrm{~mm}$. Se encontró asociación directa entre el espesor medio intimal carotídeo y la presión arterial diastólica $(r=0,193)$. Existe asociación lineal directa entre el porcentaje de grasa y la presión arterial diastólica $(r=0,283)$. Conclusión: En este estudio se encontró una relación entre el espesor medio intimal carotídeo y la presión arterial diastólica. No se evidenció relación estadística entre el espesor medio intimal carotídeo y la obesidad o el exceso de grasa corporal. Es importantísimo continuar con este tipo de estudios en la población colombiana con el fin de prevenir, desde etapas tempranas de la vida, la aparición de factores de riesgo cerebro-cardiovascular.

(c) 2015 Sociedad Colombiana de Cardiología y Cirugía Cardiovascular. Publicado por Elsevier España, S.L.U. Este es un artículo Open Access bajo la CC BY-NC-ND licencia (http:// creativecommons.org/licencias/by-nc-nd/4.0/).
\end{abstract}

\footnotetext{
* Autor para correspondencia.

Correos electrónicos: alfonsojaureguipinilla@gmail.com, alfonsojauregui@hotmail.com (A. Jáuregui).
} 


\section{KEYWORDS}

Pediatrics; cardiovascular risk factors; primary prevention; carotid arteries and ultrasound imaging

\section{Carotid intima-media thickness in school children and its relationship with cardiovascular risk factors}

\begin{abstract}
Objetive: To determine the relationship between cardiovascular risk factors and carotid intimamedia thickness in a school children population of the city of Bogotá.

Methods: Cross-sectional descriptive study including all students at the selected schools of the convenience sample. The sample gathered 94 pupils (57 girls and 37 boys) of ages $13 \pm 1.54$. Anthropometric measurements, blood pressure and ultrasound measurement of carotid intimamedia thickness were conducted. Data analysis was carried out by means of descriptive statistics, in so far the univariate and bivariate analysis was made with frequency distribution and contingency tables for qualitative variables and descriptive measures of central tendency and variability for quantitative variables.

Results: Out of the total population, $34.79 \%$ showed excess weight according to the body mass index, $28.8 \%$ registered excess body fat, $46 \%$ had high blood pressure, of which $36.8 \%$ corresponded to early arterial hypertension and 9.2\% to stage । hypertension. Average carotid intima-media thickness was $0.3163 \pm 0.040 \mathrm{~mm}$. A direct association was found between the carotid intima-media thickness and diastolic pressure $(r=0.193)$. There is a direct lineal association between the percentage of body fat and diastolic pressure $(r=0.283)$.

Conclusion: The present study found a direct association between the carotid intima-media thickness and diastolic pressure. No statistically significant evidence was found to relate carotid intima-media thickness and obesity or excess body fat. It is very important to continue this type of study in Colombian population in order to prevent, from early stages in life, the occurrence of cerebro-cardiovascular risk factors.

(c) 2015 Sociedad Colombiana de Cardiología y Cirugía Cardiovascular. Published by Elsevier España, S.L.U. This is an open access article under the CC BY-NC-ND license (http:// creativecommons.org/licenses/by-nc-nd/4.0/).
\end{abstract}

\section{Introducción}

Las enfermedades cardiovasculares son la primera causa de mortalidad en los países occidentales y en la actualidad explican por sí mismas más del $45 \%$ de las muertes en los países desarrollados ${ }^{1,2}$. En Colombia, la enfermedad coronaria es la primera causa de muerte, con una tasa de mortalidad de 64,59 por 100.000 habitantes en mayores de 50 años, seguida por la enfermedad cerebro-vascular aguda ${ }^{3}$. Se ha previsto que para el año 2020 las enfermedades crónicas no transmisibles serán las responsables de más o menos el $75 \%$ de las muertes en el mundo, de las cuales la enfermedad cardiovascular ocuparía el primer lugar ${ }^{4}$.

La presencia de arterioesclerosis, caracterizada por un engrosamiento difuso e incremento de la rigidez arterial, cambios que incluyen la capa media e íntima de las arterias, son la manifestación de la enfermedad cardiovascular. La ateroesclerosis, que es una forma de arterioesclerosis, se inicia desde la infancia, y se demuestra por la presencia de estrías grasas y engrosamiento de la pared arterial en estudios patológicos realizados en niños ${ }^{5-7}$. Los factores de progresión de la ateroesclerosis y de producción de eventos cardiovasculares involucran factores de riesgo causales como edad, tabaquismo, hipertensión arterial, hipercolesterolemia y diabetes; factores de riesgo condicionales tales como hipertrigliceridemia, concentraciones elevadas de lipoproteína (a) así como de homocisteína, e inhibidor del activador del plasminógeno (PAl-1) y fibrinógeno; y por último, factores de riesgo predisponentes como obesidad, sedentarismo, historia familiar de enfermedad arterial coronaria prematura, género masculino, alteraciones o enfermedades psíquicas, factores socioeconómicos y etnia $^{2,8-10}$.

La medición del espesor medio intimal carotídeo (EMIC) por ultrasonido se ha convertido en una herramienta útil, confiable, no invasiva, segura y económica para el diagnóstico y seguimiento de la enfermedad vascular, al ser capaz de detectar cambios estructurales como engrosamiento de las arterias carótidas y femorales, convirtiéndose así en un método diagnóstico de prevención temprana de enfermedades cerebro-cardiovasculares ${ }^{2,8,11}$.

La presencia de enfermedades cardiovasculares en la infancia es muy rara; generalmente aparece en niños con antecedentes familiares de hiperlipidemia, enfermedad renal crónica, enfermedad de Kawasaki, homocisteinuria, entre otras ${ }^{12}$. Sin embargo, sí se han identificado factores de riesgo cardiovascular como: elevación del perfil lipídico, aumento de las cifras tensionales, obesidad, sedentarismo e historia familiar de enfermedades cardiovasculares en esta población, incluso desde edades muy tempranas de la vida $^{8,13,14}$.

En Colombia, la prevalencia del exceso de peso en los niños y adolescentes entre los 5 a 17 años de edad, según la Encuesta Nacional de la Situación Nutricional (ENSIN) del año 2010 , es de $17,5 \%$, un $25,9 \%$ más que la reportada en 2005 , acompañada de sedentarismo desde tempranas etapas de la vida ${ }^{15}$. Un estudio realizado en Cartagena-Colombia con una muestra de 173 niños entre los 7 y 14 años, encontró que más de la mitad de la población presenta antecedentes familiares de enfermedad cardiovascular, así como niveles 
elevados de colesterol LDL y sedentarismo, especialmente en las niñas ${ }^{16}$. Se ha descrito que estos factores de riesgo se mantienen en la adultez ${ }^{17}$.

En el ámbito mundial se ha descrito un aumento en la prevalencia de hipertensión arterial en niños (hasta el 4\%), así como de obesidad en esta misma población ${ }^{18}$.

Algunos estudios han demostrado la utilidad de la medición del espesor medio intimal carotídeo en la detección precoz y progresión de la enfermedad vascular ${ }^{8,11}$. Se ha determinado una relación estadística entre el espesor medio intimal carotídeo y la presencia de otros marcadores para enfermedad cardiovascular en niños hipertensos o con factores de riesgo cardiovascular. El espesor medio intimal se incrementa en la adolescencia, en los niños más altos, con mayor índice de masa corporal, en presencia de niveles más altos de presión arterial sistólica y también en sujetos fumadores ${ }^{12}$. En niños obesos entre los 9 a 16 años, se ha encontrado un espesor medio intimal entre 0,37 $0,46 \mathrm{~mm}$, mayor al observado en niños sanos. Valores aún más altos se han hallado en niños con coartación de la aorta o enfermedad renal crónica $(0,54 \pm 0,05 \mathrm{~mm})$ e hiperlipidemia $(0,50 \pm 0,07 \mathrm{~mm})^{12}$. El objetivo de este trabajo fue determinar la relación entre los factores de riesgo cerebrocardiovascular y el espesor medio intimal carotídeo en una población de escolares de la ciudad de Bogotá.

\section{Materiales y Métodos}

Estudio descriptivo de corte transversal, efectuado durante el año 2014, para el cual se invitaron a participar todos los estudiantes (125) de 9 a 18 años de dos colegios de la ciudad de Bogotá seleccionados por conveniencia. Los estudiantes que ingresaron al estudio fueron aquellos que entregaron el consentimiento informado firmado por ellos y sus padres manifestando el conocimiento previo y la aceptación del estudio; la muestra estuvo conformada por un total de 94 estudiantes.

La valoración nutricional se realizó mediante la toma de peso y talla con una báscula de peso corporal electrónica (Microlife WS 100 , capacidad $150 \mathrm{~kg}$, precisión $\pm 1 \%+0,1 \mathrm{~kg}$ ) y tallímetro (precisión de $0,5 \mathrm{~cm}$ ). Estas medidas se tomaron sin zapatos, con pantaloneta y camiseta. La estatura se tomó con los pies y las rodillas juntas, talones, cara posterior de glúteos y cabeza bien adheridos al plano del tallímetro. Se ubicó una escuadra sobre el vértex y se leyó el valor de la talla en centímetros ${ }^{1}$.

El índice de masa corporal se calculó a partir de los datos de peso y talla (peso/talla ${ }^{2}$ expresado en $\mathrm{kg} / \mathrm{m}^{2}$ ). Para la clasificación nutricional se utilizaron las tablas de índice de masa corporal por edad para hombres y mujeres de 2 a 20 años, establecidas por el Centro de Estadísticas de Salud de Estados Unidos (USA National Center for Health Statistics). Los valores de índice de masa corporal clasificados como menor del percentil 5, se consideraron como bajo peso; entre el percentil 5 y menor al percentil 85 se definieron como normales; sobrepeso entre el percentil 85 y menos del 95, y obesidad en el percentil 95 o más ${ }^{1}$.

Para las medidas de los pliegues cutáneos se utilizó el calibrador tipo Langer (precisión de $1 \mathrm{~mm}$ ) y para los perímetros corporales la cinta antropométrica flexible con una escala de $10 \mathrm{~mm}$ (error $1 \mathrm{~mm}$ ). El pliegue cutáneo tricipital se midió en el punto medio entre el borde inferior del acromion y el olécranon, en la cara posterior del brazo. El pliegue cutáneo subescapular se midió a $1 \mathrm{~cm}$ distal del pliegue oblicuo generado a la altura del ángulo inferior de la escápula, en dirección de abajo hacia arriba y de adentro hacia afuera en un ángulo de $45^{\circ}$ con el plano horizontal ${ }^{1}$.

El porcentaje de grasa corporal se estimó a partir de los pliegues cutáneos, mediante las ecuaciones y la clasificación propuesta por Slaughter ${ }^{19}$.

Tríceps + subescapular: Niños: $\% \mathrm{BF}=0,783(\Sigma)+1,6$ Niñas: \%BF $=0,546(\Sigma)+9,7$

La toma de presión arterial se realizó por método auscultatorio utilizando un esfigmomanómetro previamente calibrado con brazalete pediátrico. Los estudiantes permanecieron sentados en una silla con espaldar y los pies en el suelo durante cinco minutos y se procedió a realizar la medición en el brazo derecho apoyado a la altura del corazón ${ }^{20}$.

Para la medición del espesor medio intimal carotídeo se utilizó un equipo de ultrasonido de marca «Terason Logiq» de alta resolución en modo $\mathrm{B}$ con un transductor de $10 \mathrm{MHz}$. Se realizaron seis mediciones del grosor medio intimal así: en el tercio medio de las arterias carótida común derecha y carótida común izquierda; a $10 \mathrm{~mm}$ del origen de las arterias carótidas internas y en el bulbo carotídeo de la arteria carótida derecha e izquierda. Con el paciente en decúbito supino y el cuello extendido a $45^{\circ}$ más el transductor perpendicular al vaso arterial a examinar, se evaluaron las paredes cercanas y lejanas de cada arteria ${ }^{21}$. Todas las mediciones fueron hechas por el mismo médico radiólogo con un único equipo de ultrasonido.

Los datos fueron tabulados en Microsoft Excel y el análisis estadístico se realizó con el programa SPSS versión 20 (SPSS Inc. Chicago, Illinois USA). El análisis de datos se realizó mediante estadística descriptiva, mientras que el análisis univariado y bivariado se hizo a través de tablas de distribución de frecuencias para variables cualitativas y medidas descriptivas de tendencia central para variables cuantitativas.

\section{Resultados}

La muestra correspondió a 94 estudiantes con una edad promedio de $13 \pm 1,54$ años; 57 niños y 37 niñas. De acuerdo con el índice de masa corporal, se encontraron 3 estudiantes $(3,26 \%)$ con bajo peso, $57(61,91 \%)$ con peso normal, 18 $(19,57 \%)$ con sobrepeso y $14(15,22 \%)$ con obesidad. De los sujetos con exceso de peso, las niñas representaron un 65,6\% (tabla 1).

Se observó una diferencia entre el promedio de los valores del espesor medio intimal carotídeo y el género, siendo mayor el valor en los niños $(0,3257 \mathrm{~mm})$ que el de las niñas $(0,3093 \mathrm{~mm})$.

A partir de la relación entre la medición del grosor del pliegue cutáneo del tríceps y el subescapular, se evidenció mayor porcentaje de grasa corporal en las niñas que en los niños con un $26,87 \%$ vs. $20,82 \%$, respectivamente. El valor promedio del porcentaje de grasa corporal fue de $24,51 \pm 8,54 \%$. No se observó relación lineal entre el porcentaje de grasa corporal y el espesor medio intimal carotídeo $(r=0,021)$. 
Tabla 1 Comportamiento estadístico de las variables antropométricas y de la medición ecográfica arterial en la población de estudio

\begin{tabular}{|c|c|c|c|c|}
\hline Variables & Población total ${ }^{*}$ & Niños $(n=35)^{*}$ & Niñas $(n=57)^{*}$ & $\mathrm{r}$ \\
\hline Peso (kg) & $52,83 \pm 36,87$ & $50,50 \pm 13,18$ & $54,27 \pm 45,8$ & 0,637 \\
\hline Talla $(\mathrm{cm})$ & $157,92 \pm 8,05$ & $161,306 \pm 9,14$ & $155,83 \pm 6,56$ & 0,001 \\
\hline Índice de masa corporal $\left(\mathrm{kg} / \mathrm{m}^{2}\right)$ & $19,48 \pm 3,33$ & $19,15 \pm 3,51$ & $19,68 \pm 3,23$ & 0,456 \\
\hline Porcentaje de grasa & $24,51 \pm 8,54$ & $20,82 \pm 9,31$ & $26,87 \pm 7,16$ & 0,001 \\
\hline Espesor medio intimal carotídeo & $0,3157 \pm 0,0396$ & $0,3257 \pm 0,0443$ & $0,3093 \pm 0,0351$ & 0,055 \\
\hline
\end{tabular}

Promedio \pm Desviación estándar.

En cuanto a niveles de presión arterial, el $46 \%$ de los sujetos presentó alteración de las cifras tensionales. De acuerdo con las tablas de presión arterial de la Asociación Americana de Pediatría ${ }^{20}$, 32 estudiantes (36,8\%) tuvieron prehipertensión arterial y $8(9,2 \%)$ hipertensión arterial estadio 1 . De los estudiantes con prehipertensión arterial, 25 (78,1\%) fueron niñas y $7(21,8 \%)$ niños, y del total de estudiantes con hipertensión arterial estadio 1, 4 (50\%) eran niñas y 4 (50\%) niños. No se observó diferencia entre los valores de presión arterial diastólica por género. Los valores de la tensión arterial diastólica de los sujetos estudiados se vieron afectados por el porcentaje de grasa corporal. Se observó asociación lineal directa entre el porcentaje de grasa y la presión arterial diastólica; a medida que aumenta la grasa corporal aumenta la presión arterial diastólica $(r=0,283)$.

Con relación a la medición del espesor medio intimal carotídeo se halló un valor promedio de 0,3163 $\pm 0,040 \mathrm{~mm}$. Al analizar los valores del espesor medio intimal carotídeo con la presión arterial diastólica, se observó que a medida que aumenta el valor del espesor medio intimal carotídeo, incrementa el valor de la tensión arterial diastólica $(r=0,193)$.

Entre las cifras del espesor medio intimal carotídeo en niños y niñas según peso (normal y exceso de peso), porcentaje de grasa corporal (normal y exceso) y la presión arterial (normal, prehipertensión arterial e hipertensión arterial), no se encontró una relación lineal.

\section{Discusión}

Diversos estudios han identificado la relación entre el espesor medio intimal carotídeo y la presencia de enfermedades metabólicas en la infancia ${ }^{5,8,22}$. En niños chilenos obesos sin otras alteraciones metabólicas se evidenció un aumento moderado del espesor medio intimal carotídeo ${ }^{23}$. En niños orientales de 11 a 13 años se identificó la relación entre el perímetro abdominal y el espesor medio intimal carotídeo medido por ultrasonido ${ }^{24}$.

En adolescentes obesos se ha demostrado mayor compromiso vascular por aumento del espesor medio intimal. Así mismo, se han identificado la obesidad y la presión arterial sistólica como factores de riesgo para daño vascular temprano ${ }^{23,25}$.

Sin embargo, existen otros estudios donde no se ha observado relación entre el espesor medio intimal carotídeo y la obesidad infantil ${ }^{26}$. Por tanto se ha planteado que la enfermedad vascular sólo es detectable a partir de la cuarta década de la vida ${ }^{7}$. En este trabajo se encontró una relación lineal entre el espesor medio intimal y la presión arterial diastólica; no se observaron diferencias entre el espesor medio intimal carotídeo y el género, la clasificación nutricional y el aumento de la grasa corporal.

\section{Conclusiones}

Con base en los datos encontrados en este estudio se puede deducir que el promedio del espesor medio intimal carotídeo en la población objeto, es menor que el encontrado en otras investigaciones de otros países con características similares ${ }^{7,11,23}$.

Por otra parte, a pesar de las diferentes investigaciones realizadas en el todo el mundo en la población pediátrica, aún son evidentes las falencias en la definición del grosor del espesor arterial en relación con la detección temprana de enfermedad cardiovascular en niños, así como la relación de este espesor con otros factores de riesgo cardiovascular presentes desde la infancia.

Como limitación de este estudio se considera la pérdida de información relacionada con los antecedentes heredofamiliares de la enfermedad cardiovascular, debido a la dificultad en la participación directa de los padres de familia en el momento de recolección de estos datos.

Además, se considera el sesgo del paciente voluntario dado que se incluyeron en el estudio a los niños cuyos padres aceptaron su participación.

Es importante continuar con este tipo de investigaciones encaminadas a lograr una identificación de la relación entre los factores de riesgo cardiovascular desarrollados desde la infancia y la progresión de la enfermedad cerebrocardiovascular, teniendo en cuenta estudios analíticos con el fin de contribuir en la prevención de estas enfermedades de una forma eficaz y eficiente para lo que respecta a la población colombiana.

La búsqueda de métodos diagnósticos seguros, mínimamente invasivos, con alta sensibilidad y especificidad así como económicos para el sistema de salud, constituye el reto para las investigaciones en salud con un enfoque preventivo.

\section{Responsabilidades éticas}

Protección de personas y animales. Los autores declaran que para esta investigación no se han realizado experimentos en seres humanos ni en animales. 
Confidencialidad de los datos. Los autores declaran que han seguido los protocolos de su centro de trabajo sobre la publicación de datos de pacientes.

Derecho a la privacidad y consentimiento informado. Los autores han obtenido el consentimiento informado de los pacientes y/o sujetos referidos en el artículo. Este documento obra en poder del autor de correspondencia.

\section{Conflicto de intereses}

Los autores declaran no tener de manera directa o indirecta, algún tipo de conflicto de intereses financieros, académicos o laborales que puedan poner en peligro la validez de este estudio.

\section{Agradecimientos}

Este trabajo es el resultado del proyecto de investigación MED - 1553, financiado por el Fondo de Investigaciones de la Universidad Militar Nueva Granada.

Los autores agradecen a la Vicerrectoría de Investigaciones de la Universidad Militar Nueva Granada. A los estudiantes y a sus padres de familia. A las Directivas de los Colegios participantes en este estudio. Al Doctor Alfonso Martínez Flechas, médico radiólogo, y al Doctor Guillermo Díaz, por el análisis estadístico de los datos.

\section{Bibliografía}

1. Heymsfield SB, Lohman TG, Wang Z, Going SB. Composición Corporal. $2^{\circ}$ ed. México: McGraw Hill; 2005. p.521.

2. Arnaiz P, Villarroel L, Barja S, Godoy I, Cassis B, Domínguez A. La presión arterial es un importante marcador de ateroesclerosis subclínica en niños. Rev Med Chile. 2012;140:1268-75.

3. Instituto Nacional de Salud, Observatorio Nacional de Salud, Primer Informe ONS, aspectos relacionados con la frecuencia de uso de los servicios de salud, mortalidad y discapacidad en Colombia, 2011. Bogotá (D.C): Colombia: Imprenta Nacional de Colombia; 2013.

4. Jiménez MC, Sanabria MC, Mendoza L, González R. Factores de riesgo cardiovascular en escolares y adolescentes de una comunidad rural de Amambay. Pediatr (Asunción). 2011;38(3):205-12.

5. Bel Comós J, Murillo Valles M. Obesidad y síndrome metabólico. Protoc Diagn Ter Pediatr. 2011;1:228-35.

6. Daniels SR, Greer FR, Committee on Nutrition. Estudio sistemático de los lípidos y la salud cardiovascular en la infancia. Pediatrics (Ed esp). 2008;66(1):52-62.

7. Raitakari OT, Juonala M, Kähönen $M$, Taittonen L, Laitinen T, Mäki-Torkko N, et al. Cardiovascular risk factors in childhood and carotid artery intima-media thickness in adulthood: the Cardiovascular Risk in Young Finns Study. JS.JAMA. 2003;290(17):2277-83.

8. Bravo M, Collado L, Dardanelli E, Araújo MB, Lipsich J, Moguillansky S. Medición ecográfica del espesor medio-intimal carotídeo en pacientes pediátricos con obesidad, hipercolesterolemia familiar y diabetes tipo 1. RAR. 2012;76(1):55-61.

9. Ciszek A, Bermúdez V, Leal E, Bermúdez F, Cano R, Finol F, et al. Inhibidor del activador del plasminógeno tipo 1 (PAl-1) y su relación con la aterosclerosis coronaria. Rev Lat Hipertens. 2007;2:151-6.

10. Aguilar Cordero MJ, González Jiménez E, Álvarez Ferre J, Padilla López CA, Rivas García F, Perona JS, et al. Estudio de los niveles séricos de leptina, ceruloplasmina y lipoproteína (a) como indicadores del riesgo cardiovascular en una población de adolescentes de Granada (España). Nutr Hosp. 2011;26(5): $1130-3$.

11. Simșek E, Balta H, Balta Z, Dallar Y. Childhood obesity-related cardiovascular risk factors and carotid intima-media thickness. Turk J Pediatr. 2010;52(6):602-11.

12. Litwin M, Niemirska A. Intima-media thickness measurements in children with cardiovascular risk factors. Pediatr Nephrol. 2009;24:707-19.

13. Pahkala K1, Hietalampi H, Laitinen TT, Viikari JS, Rönnemaa T, Niinikoski $\mathrm{H}$, et al. Ideal cardiovascular health in adolescence: effect of lifestyle intervention and association with vascular intima-media thickness and elasticity (the Special Turku Coronary Risk Factor Intervention Project for Children STRIP study). Circulation. 2013;127(21):2088-96.

14. Maximova K, Kuhle S, Davidson Z, Fung C, Veugelers P. Cardiovascular risk-factor profiles of normal and overweight children and adolescents: Insights From the Canadian Health Measures Survey. Canad J Cardiol. 2013;29:976-82.

15. Colombia, Ministerio de la Protección Social, Instituto Nacional de Salud, Instituto Colombiano de Bienestar Familiar. Encuesta Nacional de la Situación Nutricional en Colombia (ENSIN) 2010. Libro Digital. Bogotá: Ministerio de la Protección Social; 2010.

16. Alayón AN, Castro R, Gaviria L, Fernández M, Benítez L. Factores de riesgo cardiovascular en escolares entre 7 y 14 años en Cartagena, Colombia, 2009. Rev. Salud Pública. 2011;13(2): 196-206.

17. Greenlund KJ, Srinivasan SR, Xu JH, Dalferes E, Myers L, Pickoff A, Berenson GS. Plasma homocysteine distribution and its association with parental history of coronary artery disease in black and white children. The Bogalusa Heart Study. Circulation. 1999;99:2144-9.

18. Rab EM, Assadi F. Management of hypertension in children with cardiovascular disease and heart failure. Int J Prev Med. 2014;5 Suppl 1:S10-6.

19. Biesalski HK, Grima P. Nutrición Texto y atlas. Madrid: Editorial Médica Panamericana; 2007.

20. Chobanian AV, Bakris GL, Black HR, Cushman WC, Green LA, Izzo JL, et al., The National High Blood Pressure Education Program Coordinating Committee. The seventh report of the Joint National Committee on prevention, detection, evaluation, and treatment of high blood pressure. The JNC 7 Report. JAMA. 2003;289:2560-72

21. Espinoza A, Bagés C, Le Roy C, Castillo C. Grosor de íntima media carotídea en niños sanos por técnica ecográfica computarizada. Rev Chil Pediatr. 2011;82(1):29-34.

22. Melo X, Santa-Clara H, Pimenta NM, Carrolo M, Martins SS, Minderico CS, et al. Body composition phenotypes and carotid intima-media thickness in 11-13-year-old children. Eur J Pediatr. 2014;173(3):345-52.

23. Torreóon C, Hevia M, Ureta E, Valenzuela X, Grosor Balboa P. de la íntima-media de la arteria carótida en adolescentes obesos y su relación con el síndrome metabólico. Nutr Hosp. 2012;27(1):192-7.

24. Thomas GN, Chook P, Qiao M, Huang XS, Leong HC, Celermajer DS, Woo KS. Deleterious impact of high normal glucose levels and other metabolic syndrome components on arterial endothelial function and intima-media thickness in apparently healthy Chinese subjects: the CATHAY study. Arterioscler Thromb Vasc Biol. 2004;24(4):739-43.

25. Martínez M. Estudio de biomarcadores de inflamación y de riesgo cardiovascular en niños obesos prepúberes tesis doctoral. Córdoba: Córdoba Univ.; 2010.

26. Tounian P, Aggoun Y, Dubern B, Varille V, Guy-Grand B, Sidi D, et al. Presence of increased stiffness of the common carotid artery and endothelial dysfunction in severely obese children: a prospective study. Lancet. 2001;358(9291):1400-4. 